\title{
LINEAR SPACES, ABSOLUTE RETRACTS, AND THE COMPACT EXTENSION PROPERTY
}

\author{
JOS van der BIJL AND JAN van MILL
}

(Communicated by Doug W. Curtis)

\begin{abstract}
We formulate a "partial realization" property and prove that this property is equivalent to the compact extension property. In addition, we prove that a linear space $L$ has the compact extension property if and only if $L$ is admissible if and only if $L$ has the $\sigma$-compact extension property. This implies that for a $\sigma$-compact linear space $L$, the following statements are equivalent: (1) $L$ is an absolute retract, (2) $L$ has the compact extension property, and (3) $L$ is admissible. Finally, we prove that if there exists a linear space which is not an absolute retract then there is an admissible linear space which is not an absolute retract.
\end{abstract}

1. Introduction. All spaces under consideration are separable metric. J. Dugundji [3] proved that every locally convex linear space is an AR. The problem whether this also holds for nonlocally convex linear spaces is still open. A linear space $L$ is called admissible $[\mathbf{7}, \mathbf{8}]$ if every compact subset of $L$ admits arbitrarily small displacements into finite-dimensional linear subspaces of $L$. It is well known that every locally convex linear space is admissible (cf. [12]). Klee [8] gave examples of nonlocally convex admissible linear spaces and also [7] proved that every complete admissible linear space $L$ has the so-called compact extension property. A space $X$ has the compact (neighborhood) extension property (abbreviated $\mathrm{C}(\mathrm{N}) \mathrm{EP}$ ) provided that for every space $Y$ and every compact subspace $A$ of $Y$, every continuous function $f: A \rightarrow X$ extends over $Y$ (over some neighborhood of $A$ in $Y$ ). It is known that every space with the C(N)EP which, in addition, is compact or finite dimensional is an $\mathrm{A}(\mathrm{N}) \mathrm{R}$ [1]. Kuratowski [9] asked whether every space with the CEP is an AR. This question was answered in the negative in [10] (see also [2]). Klee $[\mathbf{7}, \mathbf{8}]$ asked whether every linear space is admissible and also whether every admissible linear space is an AR. The aim of this paper among other things is to prove that a linear space $L$ has the CEP if and only if $L$ is admissible if and only if $L$ has the " $\sigma$-compact extension property" (see $\S 3$ ). This implies that for a $\sigma$-compact linear space $L$, the following statements are equivalent: (1) $L$ is an absolute retract, (2) $L$ has the compact extension property, and (3) $L$ is admissible. We also show that if there is an example of a linear space which is not an AR, then there also exists an admissible linear space which is not an AR.

Received by the editors July 8,1987 and, in revised form, October 6, 1987.

1980 Mathematics Subject Classification (1985 Revision). Primary 55M15, 57N17.

Key words and phrases. Absolute retract, the compact extension property, linear space, admissible linear space.

The first author's research was supported by the Netherlands Organization for the Advancement of Pure Research (Z.W.O.).

The second author's research was partly supported by an NSF grant. 
The paper is organized as follows. In $\S 2$ we present a characterization of spaces with the CNEP in terms of a partial realization property and some related results. Finally, in $\S 3$ we use some of the results obtained in $\S 2$ to derive the above statements on linear spaces.

2. Partial realization. Let $X$ be a space and let $\mathscr{U}$ be an open cover of $X$. In addition, let $\mathscr{T}$ be a locally finite simplicial complex and let $\mathscr{S}$ be a subcomplex of $\mathscr{T}$ containing all the vertices of $\mathscr{T}$. A partial realization of $\mathscr{T}$ in $X$ relative to $\mathscr{S}$ and $\mathscr{U}$ is a continuous function $f:|\mathscr{S}| \rightarrow X$ such that for every $\sigma \in \mathscr{T}$ there exists $U \in \mathscr{U}$ such that $f(\sigma \cap|\mathscr{S}|) \subseteq U$. For convenience, by a quadruple $(\mathscr{T}, \mathscr{S}, f, \mathscr{U})$ we mean a partial realization of $\mathscr{T}$ in $X$ relative to $\mathscr{S}$ and $\mathscr{U}$. If $\mathscr{S}=\mathscr{T}$, then $f$ is called a full realization of $\mathscr{T}$ in $X$.

It is well known that a space $X$ is an ANR if and only if for every open cover $\mathscr{U}$ of $X$ there exists an open refinement $\mathscr{V}$ of $\mathscr{U}$ such that for every locally finite simplicial complex $\mathscr{T}$, every partial realization $(\mathscr{T}, \mathscr{S}, f, \mathscr{V})$ of $\mathscr{T}$ in $X$ extends to a full realization $\left(\mathscr{T}, \mathscr{T}, f^{*}, \mathscr{U}\right)$; this is Whitehead's Theorem and for details we refer the reader to Borsuk [1] and $\mathrm{Hu}[\mathbf{6}]$. Whitehead's Theorem is a very important tool in the process of recognizing ANRs. For that reason we are interested in a characterization of the CNEP in terms of partial realization. The following result and its proof are, of course, inspired by Whitehead's Theorem.

2.1. THEOREM. Let $X$ be a space. The following three assertions are equivalent:

(i) $X$ has the CNEP,

(ii) for every compact subset $K$ of $X$ there is a compact subset $L$ of $X$, such that for every open cover $\mathscr{U}$ of $X$ there is an open refinement $\mathscr{V}$ of $\mathscr{U}$, such that for every partial realization $(\mathscr{T}, \mathscr{S}, f, \mathscr{V})$ with $f(|\mathscr{S}|) \subseteq K$ there is a full realization $\left(\mathscr{T}, \mathscr{T}, f^{*}, \mathscr{U}\right)$ with $f^{*}(|\mathscr{T}|) \subseteq L$ and $f^{*}|| \mathscr{S} \mid=f$,

(iii) for every compact subset $K$ of $X$ there is a compact subset $L$ of $X$, such that for every open cover $\mathscr{U}$ of $X$ there is an open refinement $\mathscr{V}$ of $\mathscr{U}$, such that for every partial realization $(\mathscr{T}, \mathscr{S}, f, \mathscr{V})$ of a finite simplicial complex $\mathscr{T}$ with $f(|\mathscr{S}|) \subseteq K$ there is a full realization $\left(\mathscr{T}, \mathscr{T}, f^{*}, \mathscr{U}\right)$ with $f^{*}(|\mathscr{T}|) \subseteq L$ and $f^{*}|| \mathscr{S} \mid=f$.

ProOF. (i) $\Rightarrow$ (ii). Choose an imbedding $j: K \rightarrow Q(Q$ is the Hilbert cube). By assumption there exist an open neighborhood $V$ of $j(K)$ and a continuous function $\xi: V^{-} \rightarrow X$ such that $\xi \mid j(K)=j^{-1}$. Let $L=\xi\left(V^{-}\right)$.

Choose an arbitrary open cover $\mathscr{U}$ of $X$. Then $\xi^{-1}(\mathscr{U}) \cap V=\left\{\xi^{-1}(U) \cap V: U \in\right.$ $\mathscr{U}\}$ is an open cover of the open subspace $V$ of $Q$ and consequently there exists an open refinement $\mathscr{W}$ of this cover consisting entirely of (linearly) convex sets. The collection $\mathscr{V}_{0}=j^{-1}(\mathscr{W})$ is a cover of $K$ consisting of (relatively) open subsets of $K$, and it refines the cover $\mathscr{U} \cap K$. Now let $\mathscr{V}$ be an open refinement of $\mathscr{U}$ such that $\mathscr{V}_{0}=\mathscr{V} \cap K$. We claim that these $L$ and $\mathscr{V}$ satisfy the requirements.

Choose a locally finite simplical complex $\mathscr{T}$, a subcomplex $\mathscr{S}$ which contains all the vertices of $\mathscr{T}$ and a partial realization $f:|\mathscr{S}| \rightarrow X$ with respect to $\mathscr{V}$ such that $f(|\mathscr{S}|) \subseteq K$. By construction, for every $\sigma \in \mathscr{T}$ there is an element $W_{\sigma}$ of $\mathscr{W}$ with

$$
j \circ f(\sigma \cap|\mathscr{S}|) \subseteq W_{\sigma}
$$

By induction on the dimension of $\sigma$, a standard construction successively applying the Dugundji Extension Theorem [3 and 6], shows there is an extension $\bar{f}:|\mathscr{T}| \rightarrow Q$ 
of $j \circ f:|\mathscr{S}| \rightarrow Q$ such that $\bar{f}(\sigma)$ is contained in the convex hull of $j \circ f(\sigma \cap|\mathscr{S}|)$ for every simplex $\sigma \in \mathscr{T}$. The convexity of the $W_{\sigma}$ 's then gives us that $\bar{f}(\sigma) \subseteq W_{\sigma}$ for every $\sigma \in \mathscr{T}$. Consequently, if we define $f^{*}:|\mathscr{T}| \rightarrow X$ by $f^{*}=\xi \circ \bar{f}$, then $f^{*}$ is the required full realization.

Observe that (ii) $\Rightarrow$ (iii) is a triviality.

For (iii) $\Rightarrow(\mathrm{i})$, choose a space $Y$, a compact subset $A$ of $Y$ and a continuous function $f: A \rightarrow X$. According to [1, Chapter V, Theorem 6.2], there exist a compact AR, $Z$, and an imbedding $i: A \rightarrow Z$ such that $N=Z \backslash i(A)$ is a polytope with triangulation $\mathscr{T}=\left\{\sigma_{i}\right\}_{i=1}^{\infty}$ with $\lim _{i \rightarrow \infty} \operatorname{diam}\left(\sigma_{i}\right)=0$. Note that the polytope $N$ is metrizable, so locally finite. There is a map $\alpha: Y \rightarrow Z$ such that $i=\alpha \mid A$. Denote $f \circ i^{-1}: i(A) \rightarrow X$ by $\tilde{f}$ and identify $i(A)$ and $A$ in the sequel.

For every $p \in N^{(0)}$ pick a point $r(p) \in A$ with $d(p, r(p))=d(p, A)$. Then $r: A \cup N^{(0)} \rightarrow A$ is a retraction. Define $g: A \cup N^{(0)} \rightarrow X$ by $g=\tilde{f} \circ r$. Then $g$ clearly extends $f$. Now use (iii) to find two compacta $K_{1}$ and $K_{2}$ in $X$ such that for $K=f(A)$ we can choose $L=K_{1}$ in (iii), and for $K=K_{1}$ we can choose $L=K_{2}$. Observe that

$$
f(A) \subseteq K_{1} \subseteq K_{2} .
$$

The next step is to define three sequences, $\left(\mathscr{U}_{n}\right)_{n=0}^{\infty},\left(\mathscr{V}_{n}\right)_{n=1}^{\infty}$, and $\left(\mathscr{W}_{n}\right)_{n=1}^{\infty}$, of open covers of $X$ such that:

(1) $\mathscr{U}_{0}=\{X\}$

(2) $\mathscr{U}_{n}<\mathscr{V}_{n} \stackrel{*}{<} \mathscr{W}_{n}<\mathscr{U}_{n-1}\left(n \geq 1 ; \mathscr{U}_{n}<\mathscr{V}_{n}\right.$ means that $\mathscr{U}_{n}$ refines $\mathscr{V}_{n}$ and $\mathscr{V}_{n}{ }^{*}<\mathscr{W}_{n}$ means that $\mathscr{V}_{n}$ is a star-refinement of $\mathscr{W}_{n}$ ),

(3) for $n \geq 1$, partial realizations of finite polytopes with respect to $\mathscr{W}_{n}$ with images in $K_{1}$ can be extended to full realizations with respect to $\{B(x, 1 / 2 n): x \in$ $X$ \} with images in $K_{2}$ (if $A \subseteq X$ then $B(A, \varepsilon)$ is the open ball about $A$ with radius $\varepsilon$ of course),

(4) for $n \geq 1$, partial realizations of finite polytopes with respect to $\mathscr{U}_{n}$ with images in $f(\bar{A})$ can be extended to full realizations with respect to $\mathscr{V}_{n}$ with images in $K_{1}$.

For every $n \geq 1$, pick a Lebesgue number $\lambda_{n}>0$ for the open cover $\tilde{f}^{-1}\left(\mathscr{U}_{n}\right)$ of the compact set $A$; we may assume that $\lambda_{n} \downarrow 0$. Note that from the local finiteness of $N$ it follows that for every open $U$ in $Z$ with $A \subseteq U$ there exist only finitely many $i \in \mathbf{N}$ with $\sigma_{i} \nsubseteq U$. From this it follows that we can write $\mathscr{T}$ as a countable union of finite subcomplexes $\mathscr{S}_{n}(n \geq 0)$ of $\mathscr{T}$ with $\mathscr{S}_{n} \subseteq \mathscr{S}_{n+1}$, such that if we let $\mathscr{T}_{n}$ be the subcomplex of $\mathscr{T}$ consisting of all the simplices in $\mathscr{S}_{n} \backslash \mathscr{S}_{n-1}$ and their faces $\left(n \geq 0 ; \mathscr{S}_{-1}=\varnothing\right)$, then

(5) $\sigma \cap\left|\mathscr{S}_{n-1}\right| \neq \varnothing \Rightarrow \sigma \in \mathscr{S}_{n}(n \geq 1, \sigma \in \mathscr{T})$,

(6) $\left|\mathscr{T}_{n}\right| \subseteq B\left(A, \frac{1}{3} \lambda_{n}\right)(n \geq 1)$,

(7) $\sigma \in \overline{\mathscr{T}}_{n} \Rightarrow \operatorname{diam}(\sigma)<\frac{1}{3} \lambda_{n}(n \geq 1, \sigma \in \mathscr{T})$,

(8) $\mathscr{T}_{n} \cap \mathscr{T}_{m}=\varnothing(|n-m| \geq 2)$.

For every $n \geq 0$ put $T_{n}=\left|\mathscr{T}_{n}\right|$. It is easily seen that $T_{n} \cap T_{n+1}$ is a polytope with triangulation $\mathscr{T}_{n} \cap \mathscr{T}_{n+1}$.

Claim 1. For $n \geq 0, g \mid\left(T_{n} \cap T_{n+1}\right)^{(0)}$ is a partial realization of $\mathscr{T}_{n} \cap \mathscr{T}_{n+1}$ with respect to $\mathscr{U}_{n+1}$ having its image in $f(A)$.

Proof of Claim 1. Pick an arbitrary $\sigma \in \mathscr{T}_{n} \cap \mathscr{T}_{n+1}$. From (6) and (7) it follows that $\operatorname{diam}(\sigma)<\frac{1}{3} \lambda_{n+1}$ and $\sigma \subseteq B\left(A, \frac{1}{3} \lambda_{n+1}\right)$, so $\operatorname{diam}\left(r\left(\sigma^{(0)}\right)\right)<\lambda_{n+1}$ and there is 
a $U \in \mathscr{U}_{n+1}$ with $r\left(\sigma^{(0)}\right) \subseteq \tilde{f}^{-1}(U)$. This gives us that $g\left(\sigma^{(0)}\right) \subseteq U$. The assertion about the image is obvious of course.

From the construction of $\mathscr{U}_{n+1}$ and $K_{1}$ we conclude that for $n \geq 0$ there exists a full realization $g_{n}: T_{n} \cap T_{n+1} \rightarrow X$ with respect to $\mathscr{V}_{n+1}$ with $g_{n}\left(T_{n} \cap T_{n+1}\right) \subseteq K_{1}$ and $g_{n}(z)=g(z)\left(z \in\left(T_{n} \cap T_{n+1}\right)^{(0)}, n \geq 0\right)$. Define the polytope $R_{n}(n \geq 1)$ by

$$
R_{n}=\left(T_{n} \cap\left(T_{n+1} \cup T_{n-1}\right)\right) \cup T_{n}^{(0)}
$$

and the function $\gamma_{n}: R_{n} \rightarrow X$ by

$$
\gamma_{n}(z)= \begin{cases}g_{n-1}(z) & \left(z \in T_{n} \cap T_{n-1}\right), \\ g_{n}(z) & \left(z \in T_{n} \cap T_{n+1}\right), \\ g(z) & \left(z \in T_{n}^{(0)}\right) .\end{cases}
$$

Observe that by (8), $\gamma_{n}$ is well-defined.

Claim 2. For $n \geq 1, \gamma_{n}$ is a partial realization of $\mathscr{T}_{n}$ with respect to $\mathscr{W}_{n}$ having its image in $K_{1}$.

Proof of Claim 2. Pick an arbitrary $\sigma \in \mathscr{T}_{n}$. There exists $U_{n} \in \mathscr{U}_{n}$ with $g\left(\sigma \cap T_{n}^{(0)}\right) \subseteq U_{n}$. Using the full-realization properties of $g_{n-1}$ and $g_{n}$ we find $\gamma_{n}\left(\sigma \cap T_{n-1}\right) \subseteq V_{n}$ and $\gamma_{n}\left(\sigma \cap T_{n+1}\right) \subseteq V_{n+1}$, where $V_{n}$ and $V_{n+1}$ are unions of members of $\mathscr{V}_{n}$ and $\mathscr{V}_{n+1}$, respectively, and each such member intersects $U_{n}$. Now $\mathscr{V}_{n+1}<\mathscr{U}_{n}<\mathscr{V}_{n} \stackrel{*}{<} \mathscr{W}_{n}$ gives us the existence of a $W_{n} \in \mathscr{W}_{n}$ such that $\gamma_{n}\left(\sigma \cap R_{n}\right) \subseteq W_{n}$.

We conclude that there exists a full realization $G_{n}: T_{n} \rightarrow X$ with respect to $\{B(x, 1 / 2 n): x \in X\}$ with $G_{n} \mid R_{n}=\gamma_{n}$ and $G_{n}\left(T_{n}\right) \subseteq K_{2}$. Now define

$$
\tilde{Z}=A \cup \bigcup_{n=1}^{\infty} T_{n}
$$

and $G: \tilde{Z} \rightarrow X$ by

$$
G(z)= \begin{cases}\tilde{f}(z) & (z \in A), \\ G_{n}(z) & \left(z \in T_{n}, n \geq 1\right) .\end{cases}
$$

Claim 3. $G$ is continuous.

Proof of Claim 3. It suffices to consider $a \in \partial A$ and a sequence $\left(z_{n}\right)_{n}$ in $N$ with $z_{n} \rightarrow a$. For every $n$ choose $\sigma_{n} \in \mathscr{T}$ with $z_{n} \in \sigma_{n}$, and $p_{n} \in \sigma_{n}^{(0)}$. By some elementary observations it is possible to prove that $p_{n} \rightarrow a$ and $d\left(G\left(z_{n}\right), G\left(p_{n}\right)\right) \rightarrow$ 0 . So by continuity of $G \mid A \cup N^{(0)}=g$ we have $G\left(p_{n}\right) \rightarrow G(a)$. Consequently, $G\left(z_{n}\right) \rightarrow G(a)$ and we are done.

Finally, note that $\tilde{Y}=\alpha^{-1}(\tilde{Z})$ is a neighborhood of $A$ in $Y$ and that $F=$ $G \circ(\alpha \mid \tilde{Y}): \tilde{Y} \rightarrow X$ is an extension of $f$.

Let $X$ and $Y$ be spaces and let $f, g: Y \rightarrow X$ be continuous. If $\mathscr{U}$ is an open cover of $X$, then $d(f, g)<\mathscr{U}$ means that for every $y \in Y$ there exists $U \in \mathscr{U}$ containing both $f(y)$ and $g(y)$. In addition, we say that $f$ and $g$ are $\mathscr{U}$-homotopic, $f \sim_{\mathscr{U}} g$, if there exists a homotopy $H: Y \times I \rightarrow X$ connecting $f$ and $g$ while, moreover, for every $y \in Y$ there exists $U \in \mathscr{U}$ such that

$$
H(\{y\} \times I) \subseteq U .
$$

We shall present a few other results in the same spirit. 
2.2. THEOREM. Let $X$ have the CNEP. Then for every compact subset $K$ of $X$ there exists a compact subset $L$ of $X$ such that for every open cover $\mathscr{U}$ of $X$ there is an open refinement $\mathscr{V}$ of $\mathscr{U}$ such that if $Y$ is any space, and $f, g: Y \rightarrow X$ are continuous functions with $d(f, g)<\mathscr{V}$ and $f(Y) \cup g(Y) \subseteq K$, then there is a homotopy $H: f \sim_{\mathscr{U}} g$ with $H(Y \times I) \subseteq L$.

PROOF. Construct $L$ and $\mathscr{V}$ as in the proof of Theorem 2.1, (i) $\Rightarrow$ (ii). After this proceed as follows, adopting the notation of that proof. Choose an arbitrary space $Y$ and continuous functions $f, g: Y \rightarrow X$ and $f(Y) \cup g(Y) \subseteq K$ and $d(f, g)<\mathscr{V}$. Then $d(j \circ f, j \circ g)<\mathscr{W}$. Let $G: Y \times I \rightarrow Q$ be the straight-line homotopy from $j \circ f$ to $j \circ g$. The convexity of the members of $\mathscr{W}$ gives $G: j \circ f \sim \mathscr{W} j \circ g$, whence if we take $H=\xi \circ G$ then we find $H: f \sim \mathscr{U} g$ and $H(Y \times I) \subseteq L$.

We finish this section with the following result that will be important in $\S 3$.

2.3. THEOREM. Let $X$ have the CNEP and let $Y$ be compact. Furthermore, let $\mathscr{U}$ be an open cover of $X$ and let $f: Y \rightarrow X$ be continuous. Then there exist $a$ (finite) polyhedron $P$ and maps $g: Y \rightarrow P$ and $h: P \rightarrow X$ such that $h \circ g$ is $\mathscr{U}$-homotopic to $f$.

ProOF. Put $K=f(Y)$ and let the compact set $L$ in $X$ have the properties mentioned in Theorem 2.1(iii) for $K$. Observe that $K \subseteq L$. There are open covers $\mathscr{U}_{0}, \mathscr{U}_{1}, \mathscr{U}_{2}, \mathscr{U}_{3}$ of $X$ such that

(1) $\mathscr{U}_{3} \stackrel{*}{<} \mathscr{U}_{2}<\mathscr{U}_{1} \stackrel{*}{<} \mathscr{U}_{0}<\mathscr{U}$,

(2) any two continuous functions into $X$ with images in $L$ and which are $\mathscr{U}_{0}$-close are $\mathscr{U}$-homotopic (Theorem 2.2),

(3) partial realizations with respect to $\mathscr{U}_{2}$ having their images in $K$ can be extended to full realizations with respect to $\mathscr{U}_{1}$ having their images in $L$ (Theorem 2.1).

After this we take a finite subcover $\mathscr{V}$ of $f^{-1}\left(\mathscr{U}_{3}\right)$ of $Y$ and define $P$ to be the nerve of $\mathscr{V}$. For the map $g: Y \rightarrow P$ we take any barycentric map. For each $V \in \mathscr{V}$ let $v_{V}$ be the vertex of $P$ corresponding to $V$. Define $h^{0}: P^{(0)} \rightarrow X$ by $h^{0}\left(v_{V}\right)=f\left(y_{V}\right)$, where $y_{V} \in V$ is arbitrarily chosen. Then $h^{0}$ is a partial realization of $P$ with respect to $\mathscr{U}_{2}$. So there exists a full realization $h: P \rightarrow X$ with respect to $\mathscr{U}_{1}$ with $h(P) \subseteq L$ and $h \mid P^{(0)}=h^{0}$. An elementary verification gives us that $d(h \circ g, f)<\mathscr{U}_{0}$, whence $h \circ g \sim_{\mathscr{U}} f$.

3. Linear spaces. Let $L$ be a linear space and let $\underline{0}$ be the zero of $L$. By [4] there exists an admissible metric $d$ on $L$ satisfying the following two conditions:

(i) $d(x+z, y+z)=d(x, y)(x, y, z \in L ; d$ is translation invariant),

(ii) $d(\underline{0}, s x)<d(\underline{0}, t x)(x \in L \backslash\{\underline{0}\}, 0 \leq s<t ; d$ is strictly monotone).

In the sequel we shall supply every linear space with such a metric. The following lemma is well known and the proof is included for the sake of completeness.

3.1. LEMMA. Let $L$ be a linear space. Then $d$ has the following additional properties:

(iii) $d\left(x_{1}+y_{1}, x_{2}+y_{2}\right) \leq d\left(x_{1}, x_{2}\right)+d\left(y_{1}, y_{2}\right)\left(x_{1}, x_{2}, y_{1}, y_{2} \in L\right)$,

(iv) $d(s x, s y) \leq d(x, y)(x, y \in L, 0 \leq s \leq 1)$,

(v) if $z$ is on the straight-line segment from $x$ to $y$, then $d(x, z) \leq d(x, y)(x, y, z \in$ $L)$, 
(vi) if $S \subseteq L$ has $k$ elements, then $\operatorname{diam}(\operatorname{conv}(S)) \leq 2(k-1) \cdot \operatorname{diam}(S)(k \in \mathbf{N})$.

ProOF. Observe that (iii), (iv) and (v) are trivial. For (vi), put $S=\left\{a_{1}, \ldots, a_{k}\right\}$. Take an arbitrary $x \in \operatorname{conv}(S)$, say $x=\sum_{i=1}^{k} \lambda_{i} a_{i}$ with $\lambda_{i} \geq 0$ for every $i$ and $\sum_{i=1}^{k} \lambda_{i}=1$. Then by using (iii) and (iv) it easily follows that

$$
\begin{aligned}
d\left(x, a_{1}\right) & \left.=d\left(\sum_{i=2}^{k} \lambda_{i} a_{i},\left(1-\lambda_{1}\right) a_{1}\right)\right) \leq \sum_{i=2}^{k} d\left(\lambda_{i} a_{i}, \lambda_{i} a_{1}\right) \\
& \leq \sum_{i=2}^{k} d\left(a_{i}, a_{1}\right) \leq(k-1) \cdot \operatorname{diam}(S) .
\end{aligned}
$$

We now come to the first main result in this section. Recall from the introduction that Klee proved that every topologically complete admissible linear space has the CEP. We shall prove that the completeness assumption in this result is superfluous. So we have that admissibility implies the CEP. We shall also prove the converse.

3.2. THEOREM. Let $L$ be a linear space. Then the following statements are equivalent:

(1) $L$ is admissible,

(2) L has the CEP.

PROOF. The proof of $(1) \Rightarrow(2)$ is a more complicated version of the proof of Theorem 10 in [7]. For $\varepsilon>0$ let $D(\underline{0}, \varepsilon)=\{x \in L: d(x, \underline{0}) \leq \varepsilon\}$. Let $X$ be a space and let $f: A \rightarrow L$ be continuous, where $A \subseteq X$ is compact. We shall construct continuous functions $\phi_{n}: X \rightarrow L$ for $n=1,2, \ldots$ such that with $\tilde{\phi}_{n}=\phi_{n} \mid A$ we have

(i) $\left(f-\tilde{\phi}_{1}-\cdots-\tilde{\phi}_{n}\right)(A) \subseteq D\left(\underline{0}, 1 / 2^{n}\right)(n \geq 1)$,

(ii) $\phi_{n}(X) \subseteq D\left(\underline{0}, 1 / 2^{n-1}+1 / 2^{n}\right)(n \geq 2)$,

(iii) $\phi_{n} \mid X \backslash B(A, 1 / n) \equiv \underline{0}(n \geq 2)$.

We shall proceed by induction on $n$. There exist a finite-dimensional linear subspace $E_{1}$ of $L$ and a continuous function $\tilde{\phi}_{1}: A \rightarrow E_{1}$ such that $d\left(\tilde{\phi}_{1}, f\right)<\frac{1}{2}$. Since $E_{1}$ is an AR there is a map $\phi_{1}: X \rightarrow E_{1}$ with $\phi_{1} \mid A=\tilde{\phi}_{1}$. Suppose that $\phi_{1}, \ldots, \phi_{n-1}$ have been constructed satisfying (i), (ii) and (iii). There are a finitedimensional linear subspace $E_{n}$ of $L$ and a continuous function $\tilde{\phi}_{n}: A \rightarrow E_{n}$ such that

$$
d\left(f-\tilde{\phi}_{1}-\cdots-\tilde{\phi}_{n-1}, \tilde{\phi}_{n}\right)<1 / 2^{n} .
$$

It follows that $\tilde{\phi}_{n}(A) \subseteq D\left(\underline{0}, 1 / 2^{n-1}+1 / 2^{n}\right) \cap E_{n}$. The latter set is an AR, being a retract of $E_{n}[\overline{7}$, Proposition 8]. So there exists a continuous function $\psi_{n}: X \rightarrow D\left(\underline{0}, 1 / 2^{n-1}+1 / 2^{n}\right)$ with $\psi_{n} \mid A=\tilde{\phi}_{n}$. Now choose a Urysohn function $\alpha: X \rightarrow I=[0,1]$ which satisfies

$$
\alpha \mid A \equiv 1 \quad \text { and } \quad \alpha \mid X \backslash B(A, 1 / n) \equiv 0 .
$$

We define $\phi_{n}$ by the formula

$$
\phi_{n}(x)=\alpha(x) \cdot \psi_{n}(x) .
$$

Then $\phi_{n}$ is clearly as required (Lemma 3.1). 
Now define $\bar{f}: X \rightarrow L$ by

$$
\bar{f}(x)=\sum_{n=1}^{\infty} \phi_{n}(x) .
$$

This series converges on $X \backslash A$ by (iii) and by (i) it converges to $f$ on $A$. Finally, the continuity of $\bar{f}$ follows from (ii).

For $(2) \Rightarrow(1)$, let $K \subseteq L$ be compact and let $\varepsilon>0$. By Theorem 2.3 there are a polyhedron $P$ and maps $\xi: K \rightarrow P$ and $\eta: P \rightarrow L$ such that $d\left(\eta \circ \xi, 1_{K}\right)<\varepsilon / 2$. Let $n=\operatorname{dim} P$.

Claim 1. There exists $\delta>0$ such that whenever $A \subseteq L$ has cardinality at most $n+2$ and has diameter less than $\delta$ then $\operatorname{diam}(\operatorname{conv}(A))<\varepsilon / 2$.

Indeed, take $\delta=\varepsilon / 4(n+1)$ and apply Lemma 3.1(vi).

There is a triangulation $\mathscr{T}$ of $P$ such that for every $\sigma \in \mathscr{T}, \operatorname{diam}(\eta(\sigma))<\delta$. Let us supply $P$ with this triangulation. For every vertex $p \in P$ put $\phi(p)=\eta(p)$. The function $\phi$ may be extended linearly to a function $\psi: P \rightarrow L$.

Claim 2. $d(\eta, \psi)<\varepsilon / 2$.

Indeed, take an arbitrary $x \in P$. Take $\sigma \in \mathscr{T}$ with $x \in \sigma$. Let $V$ denote the set of vertices of $\sigma$ and put $W=\eta(V \cup\{x\})$. Then $W$ has cardinality at most $n+2$ and has diameter less than $\delta$. By Claim $1, \operatorname{conv}(W)$ has diameter less than $\varepsilon / 2$. Since $\operatorname{conv}(W)$ contains $\eta(x)$ as well as $\psi(x)$ we now obtain $d(\eta(x), \psi(x))<\varepsilon / 2$, as required.

Now put $g=\psi \circ \xi$. Then clearly $d\left(1_{K}, g\right)<\varepsilon$. Also, the range of $g$ is contained in the convex hull of a finite set, and hence in the linear hull of a finite set, i.e. in a finite-dimensional linear subspace of $L$.

Let us say that a space $X$ has the $\sigma$-compact extension property, abbreviated $\Sigma C E P$, if for every space $Y$ and for every $\sigma$-compact closed subspace $A$ of $Y$, every continuous function $f: A \rightarrow X$ can be extended over $Y$. We shall now improve Theorem 3.2 and then conclude that a $\sigma$-compact linear space is an AR if and only if it is admissible. This will be done by deriving a series of lemmas.

3.3. LemMA. Let $X$ be a space and let $A \subseteq X$ be compact. In addition, let $L$ be an admissible linear space, let $f: X \rightarrow L$ be continuous and let $\varepsilon>0$. Then there exist a finite-dimensional linear subspace $E$ of $L$ and a map $g: X \rightarrow L$ such that $d(f, g)<\varepsilon$ and $g(A) \subseteq E$.

ProOF. There are a finite-dimensional linear subspace $E$ of $L$ and a continuous function $\tilde{\phi}: A \rightarrow E$ such that $d(\tilde{\phi}, f \mid A)<\varepsilon / 2$. Since $E$ is an AR there is a continuous extension $\phi: X \rightarrow E$ of $\tilde{\phi}$. There is an open neighborhood $U$ of $A$ such that $d(f(x), \phi(x))<\varepsilon / 2$ for every $x \in U$. Let $\alpha: X \rightarrow I$ be a Urysohn function with

$$
\alpha \mid A \equiv 0 \quad \text { and } \quad \alpha \mid X \backslash U \equiv 1 .
$$

Now define $g: X \rightarrow L$ by

$$
g(x)=\alpha(x) \cdot f(x)+(1-\alpha(x)) \cdot \phi(x) .
$$

An application of Lemma 3.1(v) yields that $g$ is as required. 
3.4. LemmA. Let $X$ be a space and let $B, A \subseteq X$ be compact such that $B \subseteq A$. In addition, let $L$ be an admissible linear space, let $f: X \rightarrow L$ be continuous and let $\varepsilon>0$. If $f(B)$ is contained in a finite-dimensional linear subspace of $L$ then there exist a finite-dimensional linear subspace $E$ of $L$ and a map $g: X \rightarrow L$ such that $d(f, g)<\varepsilon, g(A) \subseteq E$ and $g|B=f| B$.

ProOF. By Lemma 3.3 there are a finite-dimensional linear subspace $E$ of $L$ and a continuous function $\phi: X \rightarrow L$ such that $\phi(A) \subseteq E$ and $d(\phi, f)<\varepsilon / 3$. Without loss of generality we may assume that $f(B) \subseteq E$. Since $E$ is an AR there is a continuous function $\tilde{f}: X \rightarrow E$ with $\tilde{f}|B=f| B$. Furthermore, there is an open neighborhood $U$ of $B$ with

$$
d(\tilde{f}(x), \phi(x))<\varepsilon / 3 \quad \text { for } x \in U .
$$

Let $\alpha: X \rightarrow I$ be a Urysohn function with

$$
\alpha \mid B \equiv 1 \quad \text { and } \quad \alpha \mid X \backslash U \equiv 0
$$

and define $g: X \rightarrow L$ by the formula

$$
g(x)=\alpha(x) \cdot \tilde{f}(x)+(1-\alpha(x)) \cdot \phi(x) .
$$

Again it follows by Lemma 3.1 that $g$ is as required.

3.5. LEMMA. Let $X$ be a $\sigma$-compact space and let $L$ be an admissible linear space. then for every continuous function $f: X \rightarrow L$ and $\varepsilon>0$ there are an $\aleph_{0^{-}}$ dimensional linear subspace $E$ of $L$ and a continuous function $g: X \rightarrow L$ such that $d(f, g)<\varepsilon$ and $g(X) \subseteq E$.

Proof. Choose compacta $X_{1}, X_{2}, \ldots$ such that

$$
X_{n} \subseteq X_{n+1} \quad \text { and } \quad X=\bigcup_{n=1}^{\infty} X_{n} .
$$

By a repeated application of the previous lemma, it is easy to construct for every $n \geq 1$ a continuous function $g_{n}: X \rightarrow L$ such that

(i) $g_{n}\left(X_{n}\right) \subseteq E_{n}$, where $E_{n}$ is a finite-dimensional linear subspace of $L(n \geq 1)$,

(ii) $g_{n}\left|X_{n-1}=g_{n-1}\right| X_{n-1}(n \geq 2)$,

(iii) $d\left(g_{n-1}, g_{n}\right)<\varepsilon / 2^{n}\left(n \geq 1, g_{0}=f\right)$.

Define $g: X \rightarrow L$ by

$$
g(x)=\lim _{n \rightarrow \infty} g_{n}(x) .
$$

Observe that for every $m$ and $x \in X_{m}$ we have $g(x)=g_{m}(x)$. By (iii), $g$ is continuous and

$$
d(f, g)<\sum_{n=1}^{\infty} \frac{\varepsilon}{2^{n}}=\varepsilon .
$$

Finally, by (i), $g(X) \subseteq E$ with $E=E_{1}+E_{2}+\cdots$.

We now come to an interesting consequence of Theorem 3.2.

3.6. THEOREM. Let $L$ be a linear space which has the CEP. Then $L$ has the $\Sigma C E P$.

ProOF. By Theorem 3.2, $(2) \Rightarrow(1)$, a linear space with the CEP is admissible. The remaining part of the proof is analogous to the proof of Theorem $3.2,(1) \Rightarrow(2)$. 
First observe that if $L^{\prime}$ is any linear space then each neighborhood of the zero $\underline{0}$ of $L^{\prime}$ of the form $D(\underline{0}, \varepsilon)=\left\{x \in L^{\prime}: d(\underline{0}, x) \leq \varepsilon\right\}$ is a retract of $L^{\prime}$ [7, Proposition 8]. The most important adjustments are now that one should use Lemma 3.5 instead of the definition of admissibility and the observation that each $\aleph_{0}$-dimensional linear space is an AR. The latter observation follows easily from Haver's Theorem [5] that every locally contractible space that is a union of countably many finite-dimensional compact is an ANR.

3.7. COROLlaRY. For a $\sigma$-compact linear space $L$ the following three conditions are equivalent:

(i) $L$ is admissible,

(ii) $L$ has the CEP,

(iii) $L$ is an $A R$.

PROOF. The equivalence (i) $\Leftrightarrow$ (ii) follows from Theorem 3.2 and the implication (iii) $\Rightarrow$ (ii) is a triviality. For (ii) $\Rightarrow$ (iii), imbed $L$ as a closed subspace in an AR $X[6$, Theorem III.2.1]. Then by Theorem 3.6, the identity $1: L \rightarrow L$ admits a continuous extension $r: X \rightarrow L$, so $L$ is a retract of $X$.

By Corollary 3.7 the question naturally arises whether admissibility of linear spaces implies the AR property. We will show that this question is the same one as the question whether every linear space is an AR.

3.8. THEOREM. If there is a linear space which is not an AR then there is an admissible linear space which is not an $A R$.

ProOF. Let $L$ be a linear space which is not an AR. Then $L \times l^{2}$ is also a linear space which is not an AR and which in addition has algebraic dimension $c$. We therefore assume without loss of generality that $L$ has algebraic dimension $c$. Put $L^{*}=L \times l^{2}$ and let $\underline{0}$ denote the zero of $L^{*}$. Define

$$
\mathscr{K}=\left\{K \subseteq L^{*}: K \text { is closed and the algebraic dimension of } K \text { is } \mathfrak{c}\right\} .
$$

Observe that $\mathscr{K}$ is nonempty since it contains $L^{*}$. Precisely such as in [13 and 11] it is possible to construct two linear subspaces $E$ and $F$ of $L^{*}$ such that:

(1) for every $K \in \mathscr{K}, K \cap E \neq \varnothing$ and $K \cap F \neq \varnothing$;

(2) $E \cap F=\{\underline{0}\}$.

We claim that $E$ is the required example. For every $x \in l^{2}$ put $L_{x}=L \times\{x\}$.

Claim 1. For every $x \in l^{2}, E \cap L_{x}$ is dense in $L_{x}$.

Let $U$ be any nonempty open subset of $L$ and take a nonempty open subset $V$ of $L$ with $V^{-} \subseteq U$. Countably many translates of $V$ cover $L$. Consequently, since $L$ has algebraic dimension $\mathfrak{c}$ and $\mathfrak{c}$ is not the sum of countably many smaller cardinals, it follows that $V$ has algebraic dimension $c$. This implies that $V^{-} \times\{x\} \in \mathscr{K}$ and consequently intersects $E$. We are done.

Claim 2. $E$ is admissible.

To this end, let $A \subseteq E$ be compact. Suppose first that the algebraic dimension of $A$ is uncountable. Then by e.g. [11] $A$ contains a linearly independent Cantor set. This Cantor set contains two disjoint Cantor sets which by (1) both have to intersect $F$. This contradicts (2) since $A \subseteq E$. We conclude that the algebraic dimension of $A$ is countable from which it follows that $A$ is contained in an $\aleph_{0^{-}}$ dimensional linear subspace $H$ of $E$. Since $H$ is an AR (again by Haver's Theorem), 
$H$ is admissible e.g. by Corollary 3.7. So $A$ admits arbitrarily small displacements into finite-dimensional linear subspaces of $H$ and hence of $E$.

Claim 3. $E$ is not an ANR.

To the contrary, assume that $E$ is an ANR. Since $E$ is contractible, the Borsuk Homotopy Extension Theorem implies that $E$ is an AR [1, Theorem IV.9.1]. By a similar technique as in $[\mathbf{1 0}]$ we shall derive a contradiction. We may assume that $L^{*}$. is a subspace of the endface $W=\{1\}_{1} \times[-1,1]_{2} \times[-1,1]_{3} \times \cdots$ of the Hilbert cube $Q=\prod_{n=1}^{\infty}[-1,1]_{n}$. The following result is well known and its proof is included for completeness sake.

Subclaim 3.1. For every subset $P$ of $W,(Q \backslash W) \cup P$ is an AR.

Proof. Indeed, let $Z$ be any space, let $A \subseteq Z$ be closed and let $f: A \rightarrow(Q \backslash W) \cup P$ be continuous. It is clear that there is a deformation $H: Q \times I \rightarrow Q$ such that $H_{0}=1$ and $H(Q \times(0,1]) \subseteq Q \backslash W$ (e.g. define $H$ by $\left.H\left(\left(x_{1}, x_{2}, \ldots\right), t\right)=\left((1-t) x_{1}, x_{2}, \ldots\right)\right)$. Since $Q$ is an AR there is a continuous extension $F: Z \rightarrow Q$. Now define $\bar{f}: Z \rightarrow$ $(Q \backslash W) \cup P$ by the formula

$$
\bar{f}(z)=H_{d(z, A)}(F(z)) .
$$

This completes the proof of the subclaim.

Put $T=(Q \backslash W) \cup E$. Then $E$ is closed in $T$ and $E$ is an AR, so there is a retraction $r: T \rightarrow E \subseteq W$. There is a $G_{\delta}$-subset $S$ of $Q$ which contains $T$ such that $r$ can be extended to a continuous function $s: S \rightarrow W$. Then $S \cap L^{*}$ is a $G_{\delta}$-subset of $L^{*}$ containing $E$. Write $L^{*} \backslash S$ as $\bigcup_{n=1}^{\infty} K_{n}$ with each $K_{n}$ closed in $L^{*}$. Observe that $K_{n} \cap E=\varnothing$ for every $n$. From (1) we conclude that all the $K_{n}$ 's have algebraic dimension less than $\mathfrak{c}$ which, again by the fact that $\mathfrak{c}$ is not the sum of countably many smaller cardinal numbers, implies that $L^{*} \backslash S$ has algebraic dimension less than $c$. Now consider the projection $\pi: L^{*} \rightarrow l^{2}$. Since $\pi$ is linear, the algebraic dimension of $\pi\left(L^{*} \backslash S\right)$ is also less than c. Since the algebraic dimension of $l^{2}$ is certainly $\mathfrak{c}$, there exists $x \in l^{2}$ missing $\pi\left(L^{*} \backslash S\right)$. Now consider $L_{x}=L \times\{x\}$. Then $L_{x} \subseteq S$ and by Claim $1, E \cap L_{x}$ is dense in $L_{x}$. Since $s\left|E \cap L_{x}=r\right| E \cap L_{x}$, and since $L_{x}$ is contained in the domain of $s$, we conclude that $s \mid L_{x}$ is the identity. Now put $T^{\prime}=T \cup L_{x}$. Then by Subclaim 3.1, $T^{\prime}$ is an AR and the function $s \mid T^{\prime}: T^{\prime} \rightarrow E \cup L_{x}$ is a retraction. Consequently, $E \cup L_{x}$ is an AR and since $L_{x}$ is clearly a retract of $E \cup L_{x}$ this implies that $L_{x}$, and hence $L$, is an AR. Contradiction.

\section{REFERENCES}

1. K. Borsuk, Theory of retracts, PWN, Warsaw, 1967.

2. D. W. Curtis and J. van Mill, The compact extension property, Proc. Sixth Prague Topology Sympos. Hildermann, Berlin, 1988, pp. 115-119.

3. J. Dugundji, An extension of Tietze's Theorem, Pacific J. Math. 1 (1951), 353-367.

4. M. Eidelheit and S. Mazur, Eine Bemerkung über die Räume von Typus (F), Studia Mat. 7 (1938), 159-161.

5. W. E. Haver, Locally contractible spaces that are absolute neighborhood retracts, Proc. Amer. Math. Soc. 40 (1973), 280-284.

6. S. T. Hu, Theorem of retracts, Wayne State Univ. Press, Detroit, 1965.

7. V. Klee, Shrinkable neighborhoods in Hausdorff linear spaces, Math. Ann. 141 (1960), 281-285.

8. L_ Leray-Schauder theory without local convexity, Math. Ann. 141 (1960), 286-296.

9. K. Kuratowski, Sur quelques problèmes topologiques concernant le prolongement des fonctions continus, Colloq. Math. 2 (1951), 186-191. 
10. J. van Mill, Another counterexample in ANR theory, Proc. Amer. Math. Soc. 97 (1986), 136138.

11. _ Domain invariance in infinite-dimensional linear spaces, Proc. Amer. Math. Soc. 101 (1987), 173-180.

12. M. Nagumo, Degree of mapping in convex linear topological spaces, Amer. J. Math. 73 (1951), 497-511.

13. R. Pol, An infinite-dimensional pre-Hilbert space not homeomorphic to its own square, Proc. Amer. Math. Soc. 90 (1984), 450-454.

SUBFACULTEIT WISKUNDE EN INFORMATICA, VRIJE UNIVERSITEIT, DE BOELELAAN 1081, 1081 HV AMSTERDAM, THE NETHERLANDS 